\title{
The Impact of Allergic Rhinitis on Symptom Improvement in Pediatric Patients After Adenotonsillectomy
}

\author{
Dong-Jun Lee* $\cdot$ Young-Jun Chung* $\cdot$ Yeon-Jun Yang $\cdot$ Ji-Hun Mo \\ Department of Otorhinolaryngology, Dankook University College of Medicine, Cheonan, Korea
}

Objectives. It is well known that allergic rhinitis (AR) has positive association with adenotonsillectomy. However, the impact of AR on symptom improvement after adenotonsillectomy is not well documented. Hence, we aimed to evaluate the effect of AR on the symptom improvement after adenotonsillectomy between AR and nonallergic patients.

Methods. A retrospective analysis was performed on 250 pediatric patients younger than 10 years old who received adenotonsillectomy from June 2009 to June 2014 in a tertiary referral hospital. All patients underwent skin prick test or multiple allergen simultaneous test (MAST) before surgery and classified into AR group and control group. Obstructive and rhinitis symptoms including snoring, mouth breathing, nasal obstruction, rhinorrhea, itching, and sneezing were evaluated before and 1 year after surgery using questionnaire and telephone survey.

Results. AR group was 131 and control group was 119, showing higher prevalence (52.4\%) of AR among adenotonsillectomized patients. Both groups showed dramatic improvement of symptoms such as snoring and mouth breathing after surgery (all $P<0.05$ ). However, AR group showed significantly less improvement than control group in snoring, mouth breathing, nasal obstruction, and rhinorrhea (all $P<0.05$ ). Multivariate analysis showed that preoperative mouth breathing and snoring were dependent on tonsil grade and postoperative symptoms were mainly dependent on presence of AR. Nasal obstruction was dependent on tonsil grade and presence of AR preoperatively and presence of AR postoperatively. These suggest the importance of AR as a risk factor for mouth breathing, snoring, and nasal obstruction.

Conclusion. AR has positive association with adenotonsillectomy and not only allergic symptoms but also obstructive symptoms such as snoring and mouth breathing improved less in AR group than control group. Hence, patients with AR should be monitored for long-term basis and more carefully after adenotonsillectomy.

Keywords. Adenoidectomy; Allergic Rhinitis; Child; Tonsillectomy

\section{INTRODUCTION}

The most common cause of upper airway obstruction in children is adenotonsillar hypertrophy and it is usually treated with

- Received May 5, 2017

Revised July 5, 2017

Accepted July 13, 2017

- Corresponding author: Ji-Hun Mo

Department of Otorhinolaryngology, Dankook University College of

Medicine, 119 Dandae-ro, Dongnam-gu, Cheonan 31116, Korea

Tel: +82-41-550-3933, Fax: +82-41-556-1090

E-mail: jihunmo@gmail.com

*The first two authors contributed equally to this study. adenotonsillectomy. Adenotonsillectomy can resolve upper airway obstruction in about $80 \%-90 \%$ of children. However, since many children underwent adenotonsillectomy, the remaining $10 \%$ to $20 \%$ of patients were still large numbers and they complained of obstructive symptoms such as snoring and nasal obstruction even after adenotonsillectomy during their long follow-up periods [1].

Several studies have reported the postoperative outcomes of adenotonsillectomy; however, most of them are short-term outcomes rather than long-term ones [2]. It has been reported that in some patients, airway problems persist or recur over longterm follow-up, although the causes for this is not clear yet [3].

Copyright $\odot 2018$ by Korean Society of Otorhinolaryngology-Head and Neck Surgery.

This is an open-access article distributed under the terms of the Creative Commons Attribution Non-Commercial License (http://creativecommons.org/licenses/by-nc/4.0)

which permits unrestricted non-commercial use, distribution, and reproduction in any medium, provided the original work is properly cited. 
In addition, the relationship between adenotonsillectomy and postoperative airway symptoms has not been clarified till date. Thus, the successful maintenance of improved airway status after adenotonsillectomy in children remains partially challenging for otolaryngologists.

Although allergic rhinitis (AR) is an important risk factor for tonsil and adenoid hypertrophy [4] and also a major factor affecting the quality of life of children, there have been few studies on the allergic status in children after adenotonsillectomy. Moreover, there is a paucity of data indicating that AR may influence the postoperative outcomes of adenotonsillectomy.

Therefore, we aimed to compare the improvement in symptoms after adenotonsillectomy between patients with and without $\mathrm{AR}$, and to reveal the factors affecting the pre- and postoperative improvements in symptoms.

\section{MATERIALS AND METHODS}

\section{Subject}

Two hundred and eighty-two patients under the age of 10 years, who underwent adenotonsillectomy by single surgeon from June 2009 to June 2014 in a tertiary referral hospital, were initially recruited in this study. Among them, 32 patients whose chief complaint was other than obstructive symptoms (frequent tonsillitis, 23; frequent otitis media, 5; and rhinosinusitis, 4 cases, respectively) were excluded. In addition, 3 patients with subclinical allergy were also excluded. Their mean age was $6.0 \pm 2.2$ years and male: female ratio was 140:110.

A retrospective chart review was performed to collect data. All the subjects underwent skin prick test or multiple allergen simultaneous test (MAST) and they completed questionnaires. AR was confirmed by the presence of the typical symptoms and positive skin prick test or MAST. Subjects were classified into AR group and control group depending on the presence of AR.

\section{Symptom questionnaires}

Preoperative symptoms including mouth breathing, snoring, na-

\section{H I G H L I G G H T S}

- Allergic rhinitis (AR) is more prevalent in adenotonsillectomized children than general population.

- Patients with AR had more severe symptoms including snoring, nasal obstruction and rhinorrhea preoperatively.

- Both obstructive symptoms such as snoring, mouth breathing, and allergic symptoms improved significantly less in patients with AR.

- Hence, patients with AR should be monitored for long-term basis and more carefully after adenotonsillectomy due to remaining symptoms. sal obstruction, rhinorrhea, itching, and sneezing were evaluated using a visual analogue scale (VAS) from 0 (no symptom) to 10 points (very severe symptoms). For postoperative symptoms, a telephonic survey was conducted to investigate any change in symptoms at least one year after surgery. Primary caregivers completed preoperative and postoperative questionnaires in most cases.

\section{Preoperative physical examination}

Adenotonsillar enlargement was determined physical examination and cephalometry. Tonsillar hypertrophy was graded from 0 to 4 according to the Friedman staging system [5]. Adenoid hypertrophy was graded using the adenoid/nasopharynx ratio, which is well described in the literature; grade 1 denotes $0 \%$ to $25 \%$ hypertrophy, grade 2 denotes $25 \%$ to $50 \%$, grade 3 denotes $50 \%$ to $75 \%$, and grade 4 denotes $75 \%$ to $100 \%$ hypertrophy [6].

\section{Skin prick test and multiple allergen simultaneous test}

Skin prick tests were performed with 50 common aeroallergens including house dust mite, grass, tree, weed, feathers, cockroach, cat, dog, and moulds. All skin test and test reading were performed by experienced personnel.

For each allergen, we measured the largest diameter of the wheal and the diameter orthogonal to it; we then calculated the mean. A positive reaction was defined as a mean wheal diameter more than or equal to that of histamine (positive control). All the saline controls were negative. Patients were considered allergic if they had at least one positive skin prick test to any of the allergens tested [7]. When patients were too young to perform skin prick test or taking medications interfering with the skin prick test reaction, MAST was performed as follows; Patient serum was added to MAST pette chambers, which contained 30 kinds of allergens. After 2 hours of incubation and washes, enzyme-tagged anti-immunoglobulin E (IgE) was added. After another 2 hours of incubation and washes, luminescent reagent was added. After 10 minutes of incubation, the results were interpreted as class 0-4, using MAST OPTIGEN luminometer (Hitachi Chemical Diagnostics Inc., Mountain View, CA, USA). Class $\geq 2$ was interpreted as positive [8].

\section{Data evaluation and statistical analysis}

Paired comparison $t$-test was used to compare symptoms before and after surgery. Symptomatic changes were compared between AR and control groups. Student $t$-test was used to analyze the differences between the two groups. The association between symptoms (mouth breathing, snoring, nasal obstruction, rhinorrhea, itching, and sneezing) and related factors including sex, asthma, allergy, adenoid grade, and tonsil grade was analyzed using logistic regression analysis. All the parametric results are expressed as mean \pm standard deviations. Statistical significance was assumed at $P<0.05$ for all the parameters. 


\section{RESULTS}

\section{Demographic data}

Among 250 patients, 131 had AR (AR group) and 119 did not have AR (control group). Mean ages were 6.1 \pm 2.1 years and $5.9 \pm 2.1$ years in the AR and control group, respectively and mean follow-up periods were similar in both groups $(25.2 \pm 10.2$ months and 26.4 \pm 11.3 months, respectively). Only one child in the AR group had asthma out of the 250 patients (Table 1).

\section{Preoperative symptoms}

The most severe symptoms in the AR group was nasal obstruction (average symptom score, $8.52 \pm 1.61$ ), followed by snoring, rhinorrhea, itching, sneezing, and mouth breathing with average symptom scores of $7.93 \pm 1.62,7.83 \pm 2.52,7.81 \pm 3.31,7.32 \pm$ 3.24 , and $7.02 \pm 2.51$, respectively. Control group complained of mouth breathing as the most severe symptom followed by nasal obstruction, snoring and rhinorrhea. Itching and sneezing symptom scores were significantly lower in control group $(P<0.01)$. Most of the symptom scores except mouth breathing were sig-

Table 1. Demographic data of the patients

\begin{tabular}{lccc}
\hline Demographic & $\begin{array}{c}\text { AR group } \\
(n=131)\end{array}$ & $\begin{array}{c}\text { Control group } \\
(n=119)\end{array}$ & P-value \\
\hline Age (yr) & $6.1 \pm 2.1$ & $5.9 \pm 2.1$ & NS \\
Sex (male:female) & $78: 53$ & $62: 57$ & 0.023 \\
Tonsil grade & & & - \\
I & 21 & 17 & \\
II & 42 & 40 & - \\
III & 56 & 43 & \\
IV & 12 & 19 & \\
Adenoid grade & & & \\
I & 25 & 18 & \\
II & 39 & 34 & NS \\
III & 54 & 51 & NS \\
IV & 13 & 16 & \\
Associated condition & & $26.4 \pm 11.3$ & \\
Asthma & $25.2 \pm 10.2$ & & \\
Follow-up period (mo) & 25 & & \\
\hline
\end{tabular}

Values are presented as number or mean \pm standard deviation. AR, allergic rhinitis; NS, nonsignificant.

Table 2. Preoperative symptoms of AR group and control group

\begin{tabular}{lccc}
\hline \multirow{2}{*}{ Symptom } & \multicolumn{3}{c}{ Preoperative VAS score } \\
\cline { 2 - 4 } & $\begin{array}{c}\text { AR group } \\
(\mathrm{n}=131)\end{array}$ & $\begin{array}{c}\text { Control group } \\
(\mathrm{n}=119)\end{array}$ & $P$-value \\
\hline Mouth breathing & $7.02 \pm 2.51$ & $7.51 \pm 2.32$ & $\mathrm{NS}$ \\
Snoring & $7.93 \pm 1.62$ & $6.12 \pm 2.43$ & 0.032 \\
Nasal obstruction & $8.52 \pm 1.61$ & $6.42 \pm 2.52$ & 0.029 \\
Rhinorrhea & $7.83 \pm 2.52$ & $5.31 \pm 2.85$ & 0.027 \\
Itching & $7.81 \pm 3.31$ & $1.52 \pm 2.13$ & 0.003 \\
Sneezing & $7.32 \pm 3.24$ & $1.10 \pm 1.53$ & 0.003 \\
\hline
\end{tabular}

AR, allergic rhinitis; VAS, visual analogue scale; NS, nonsignificant. nificantly higher in AR group than in control group (Table 2).

\section{Postoperative symptom improvement}

Symptoms including mouth breathing, snoring, rhinorrhea, and nasal obstruction were significantly improved postoperatively in both groups. Symptom improvements in control group was significantly higher than those in the AR group (all $P<0.05)$ (Fig. 1).

The itching symptom had statistically significant improvement in the AR group, but not in the control group before and after surgery; the degree of symptom improvement was greater in the AR group. Sneezing did not show any statistically significant improvement before and after surgery, and there was no statistically significant difference in symptom change between the two groups (Fig. 1).

The influence of the size of the tonsils and adenoids on symptom improvement

We then analyzed postoperative symptom improvement according to the grade of the tonsils and adenoids, and compared them between the two groups. Obstructive symptoms like mouth breathing and snoring were significantly improved after surgery in both groups and they were dependent on the Friedman tonsil
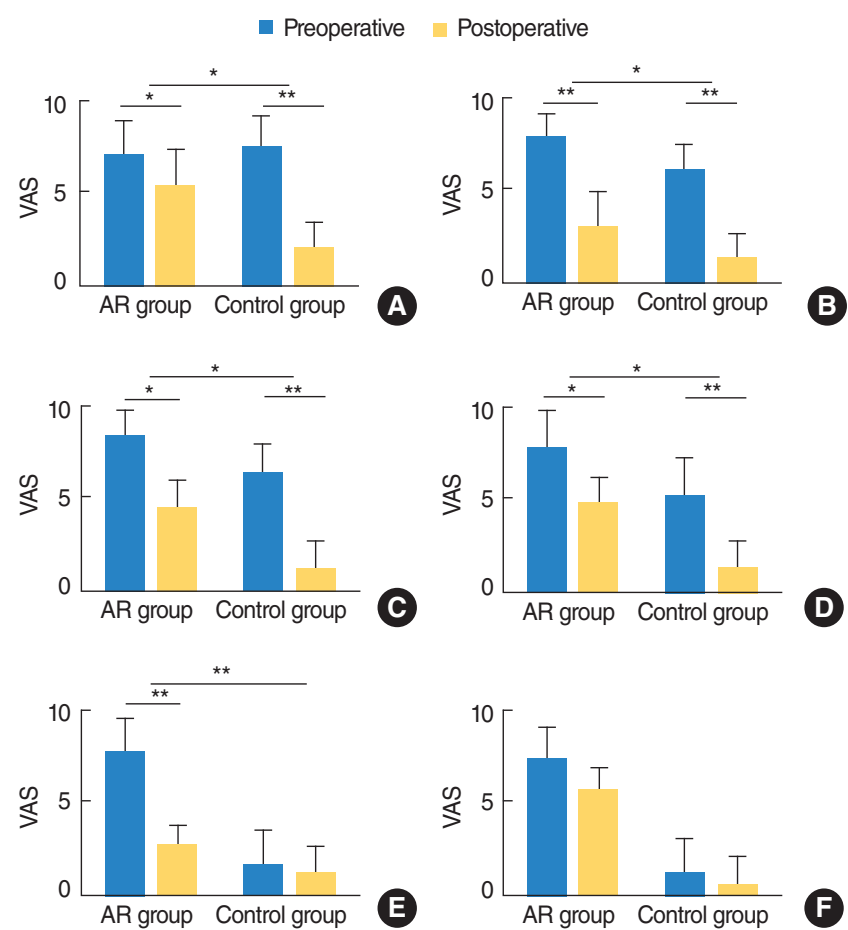

Fig. 1. Postoperative symptom improvement after adenotonsillectomy. (A) Mouth breathing, (B) snoring, (C) nasal obstruction, (D) rhinorrhea, $(E)$ itching, and $(F)$ sneezing. Both allergic rhinitis $(A R)$ group and control group showed improvement of symptoms in mouth breathing, snoring, nasal obstruction, and rhinorrhea. Control group showed significantly greater improvement in symptoms except itching and sneezing than AR group. VAS, visual analogue scale. ${ }^{\star} P<0.05 .{ }^{\star} * P<0.01$ 

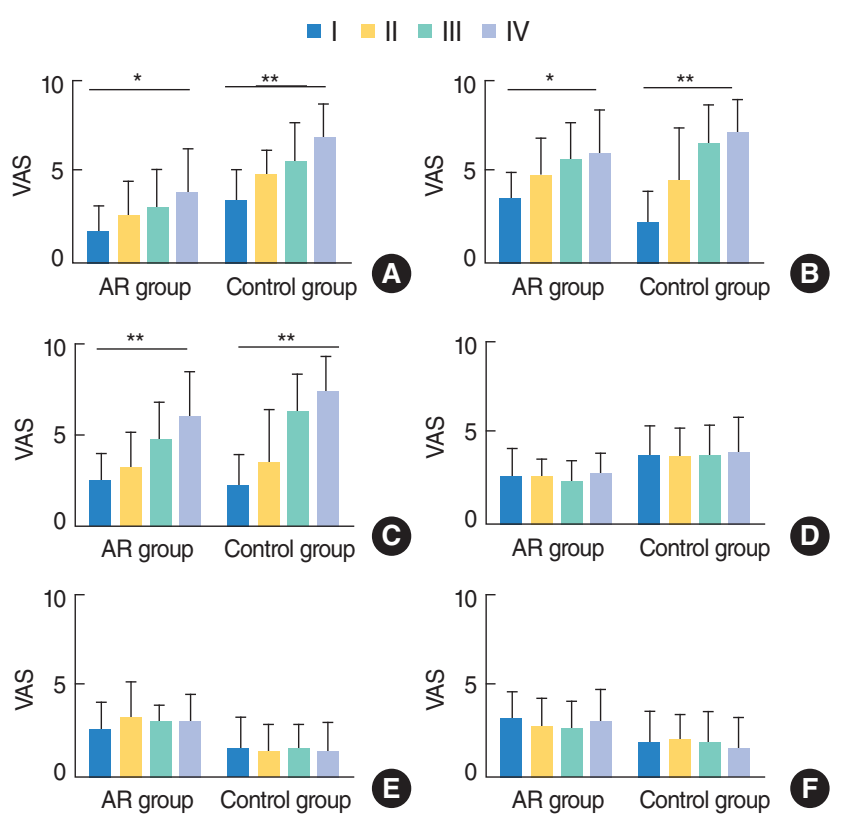

Fig. 2. The dependency of symptom changes on the Friedman tonsil grade. (A) Mouth breathing, (B) snoring, (C) nasal obstruction, (D) rhinorrhea, $(E)$ itching, and $(F)$ sneezing. Obstructive symptoms like mouth breathing and snoring were significantly dependent on Friedman tonsil grade in both groups. Nasal obstruction also showed dependency on Friedman tonsil grade in both groups, however rhinorrhea, itching, and sneezing did not show any dependency on Friedman tonsil grade. VAS, visual analogue scale; AR, allergic rhinitis. ${ }^{\star} P<0.05$. ${ }^{\star *} P<0.01$.

grade (all $P<0.05$ ) (Fig. 2). The dependency on the Friedman tonsil grade was more prominent in control group when compared with that of AR group. Postoperative improvement of nasal obstruction was also dependent on Friedman tonsil grade in both groups. However, improvement in rhinorrhea, itching, and sneezing was not dependent on the tonsil grade, as expected.

However, the degree of adenoid enlargement did not affect the improvement in either obstructive or nonobstructive symptoms after surgery (Fig. 3).

Analysis of association between symptom and variable factor Lastly, logistic regression analysis was performed to investigate the associated factors that determine the pre- and postoperative symptoms (mouth breathing, snoring, nasal obstruction, and rhinorrhea). Multiple variables such as sex, asthma, AR, tonsil grade, and adenoid grade were used. Preoperative obstructive symptoms such as mouth breathing and snoring were associated mainly with the Friedman tonsil grade; However, postoperative symptoms were significantly associated with AR $(P<0.001)$. Nasal obstruction was associated with both AR and Friedman tonsil grade before surgery, and significantly associated with AR after surgery $(P<0.001)$, Rhinorrhea was associated with AR before and after surgery (Table 3 ).
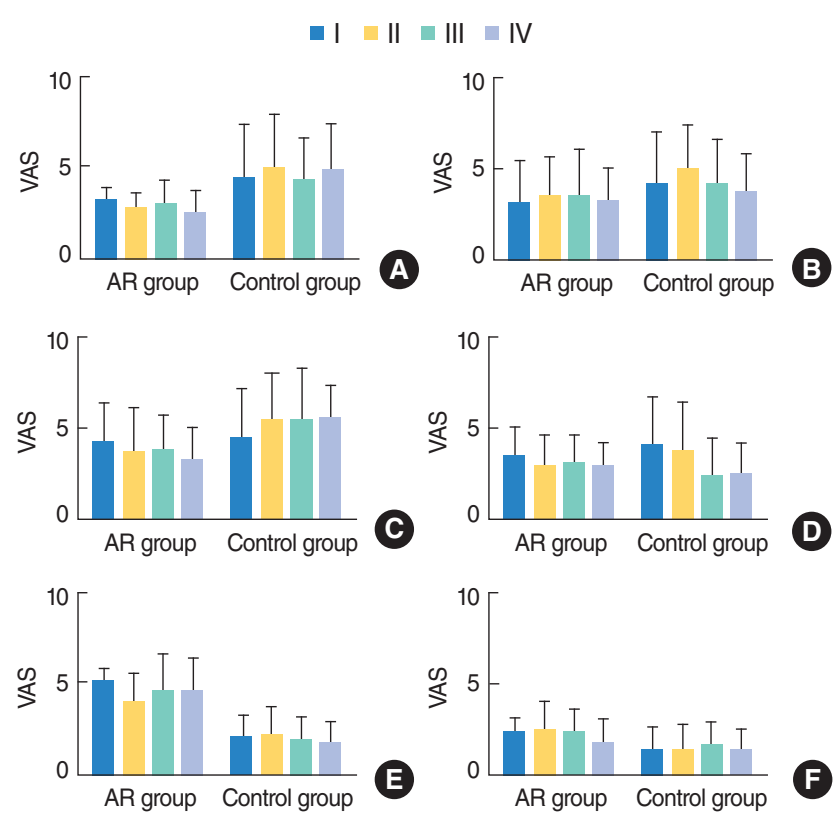

Fig. 3. The dependency of symptom changes on the adenoid grade. (A) Mouth breathing, (B) snoring, (C) nasal obstruction, (D) rhinorrhea, $(E)$ itching, and (F) sneezing. All the symptoms did not show any dependency on adenoid grade. VAS, visual analogue scale; AR, allergic rhinitis.

\section{DISCUSSION}

Adenotonsillectomy is the primary therapeutic approach for upper airway problems in children $[9,10]$. It could resolve upper airway obstruction in most cases. However, it does not always lead to complete remission of the upper airway obstructive symptoms and residual mild airway problems were found after adenotonsillectomy in more than one third of the patients $[11,12]$. These findings imply that other factors could be responsible for the postoperative remained symptoms.

Among several factors other than adenotonsillar hypertrophy, AR was considered to influence upper airway obstruction significantly [13], and previous studies showed that by the age of six, $42 \%$ of the children had AR [14]. Considering the higher prevalence of AR in children, the exact changes in symptoms after adenotonsillectomy should be analyzed according to the presence of AR.

In this study, we showed that patients with AR had more severe obstructive and rhinitis symptoms preoperatively and even after adenotonsillectomy. In addition, although adenotonsillectomy had improved symptoms in both AR and control groups, the degrees of symptom improvement were significantly lower in AR group in most of the symptoms except itching and sneezing. Therefore, these findings suggest the importance of AR management in patients with AR even after adenotonsillectomy.

AR was known to be an important factor associated with ab- 
Table 3. Association of symptoms with multiple variables with logistic regression analysis

\begin{tabular}{|c|c|c|c|c|c|c|}
\hline \multirow{2}{*}{ Variable } & \multicolumn{3}{|c|}{ Preoperative } & \multicolumn{3}{|c|}{ Postoperative } \\
\hline & $\beta$ & $95 \% \mathrm{Cl}$ & $P$-value & $\beta$ & $95 \% \mathrm{Cl}$ & $P$-value \\
\hline \multicolumn{7}{|l|}{ Mouth breathing } \\
\hline Sex & 1.06 & $0.54-2.08$ & 0.852 & 1.48 & $0.75-2.93$ & 0.252 \\
\hline $\mathrm{AR}$ & 0.72 & $0.37-1.41$ & 0.341 & 23.06 & $9.51-55.88$ & $<0.001^{*}$ \\
\hline Asthma & 1.22 & $0.18-8.18$ & 0.833 & 1.16 & $0.21-6.42$ & 0.863 \\
\hline Tonsil grade & 6.31 & $2.59-15.38$ & $<0.001^{*}$ & 0.63 & $0.27-1.46$ & 0.282 \\
\hline Adenoid grade & 0.66 & $0.34-1.27$ & 0.212 & 0.84 & $0.42-1.67$ & 0.631 \\
\hline \multicolumn{7}{|l|}{ Snoring } \\
\hline Sex & 1.21 & $0.43-2.18$ & 0.732 & 1.02 & $0.49-2.12$ & 0.261 \\
\hline AR & 0.91 & $0.27-1.72$ & 0.291 & 11.02 & $3.21-21.8$ & $<0.001^{*}$ \\
\hline Asthma & 0.87 & $0.28-1.48$ & 0.736 & 1.12 & $0.12-5.21$ & 0.751 \\
\hline Tonsil grade & 5.12 & $1.59-11.21$ & $<0.001^{*}$ & 0.52 & $0.12-1.53$ & 0.232 \\
\hline Adenoid grade & 0.71 & $0.32-1.15$ & 0.323 & 0.71 & $0.35-1.40$ & 0.325 \\
\hline \multicolumn{7}{|l|}{ Nasal obstruction } \\
\hline Sex & 0.85 & $0.21-2.10$ & 0.722 & 0.97 & $0.35-2.93$ & 0.312 \\
\hline AR & 4.12 & $0.37-10.40$ & $<0.001^{*}$ & 10.62 & $3.21-21.48$ & $<0.001^{*}$ \\
\hline Asthma & 0.71 & $0.29-1.51$ & 0.293 & 0.48 & $0.19-6.42$ & 0.763 \\
\hline Tonsil grade & 5.25 & $1.49-13.28$ & $<0.001^{*}$ & 0.71 & $0.27-3.46$ & 0.425 \\
\hline Adenoid grade & 0.59 & $0.21-1.37$ & 0.515 & 0.56 & $0.12-6.48$ & 0.236 \\
\hline \multicolumn{7}{|l|}{ Rhinorrhea } \\
\hline Sex & 0.71 & $0.32-1.52$ & 0.752 & 1.48 & $0.75-2.93$ & 0.253 \\
\hline AR & 9.21 & $2.23-19.31$ & $<0.001^{*}$ & 10.01 & 2.53-21.11 & $<0.001^{*}$ \\
\hline Asthma & 0.58 & $0.20-1.52$ & 0.635 & 0.68 & $0.11-1.91$ & 0.565 \\
\hline Tonsil grade & 1.23 & $0.41-3.21$ & 0.792 & 0.53 & $0.21-8.67$ & 0.371 \\
\hline Adenoid grade & 0.70 & $0.29-1.25$ & 0.353 & 0.62 & $0.22-1.87$ & 0.613 \\
\hline
\end{tabular}

$\mathrm{Cl}$, confidence interval; $\mathrm{AR}$, allergic rhinitis.

${ }^{*} P$-values were statistically significant.

errant facial growth. Remaining nasal obstruction as well as obstructive symptoms can cause the craniofacial morphometric changes [15]. Nasal obstruction can also induce neuromuscular changes and these changes extend beyond the period of obstruction and remain after the original stimulus for neuromuscular changes has been removed [16]. Taken these findings together, they suggest that postoperative AR care should be performed to prevent complications of nasal obstruction.

We performed logistic regression analysis to investigate the determining factor for each symptom (mouth breathing, snoring, nasal obstruction, rhinorrhea) and multiple factors including sex, asthma, AR, tonsil grade, and adenoid grade were analyzed as variables.

Friedman tonsil grade was an important determining factor for obstructive symptoms such as mouth breathing or snoring before surgery, which could be easily deducible by simple thought. The Friedman tonsil grade also affected nasal obstruction before adenotonsillectomy. Usually, tonsillar hypertrophy obstructs nasopharyngeal airway and results in mouth breathing with the aid of adenoid hypertrophy. The nasopharyngeal obstruction by tonsillar hypertrophy might affect the preoperative nasal obstruction symptom.

In contrast to Friedman tonsil grade, AR affected symptoms both before and after surgery. AR was associated with nasal ob- struction and rhinorrhea preoperatively and postoperatively, and was also associated with mouth breathing and snoring postoperatively, suggesting the importance of AR as a risk factor for obstructive symptoms such as snoring and mouth breathing even after surgery. One previous study reported similar results that AR was a risk factor that worsened the patient's postoperative symptoms, which is consistent with our data [17]. Other studies also showed the similar results that AR is an important factor in maintaining the improvements after adenotonsillectomy. Recent studies have reported that sex, age, and adiposity do not affect the postoperative symptoms after adenotonsillectomy, and that $\mathrm{AR}$ is the major risk factor for deterioration of symptoms in the long term after adenotonsillectomy $[17,18]$.

One thing that is noteworthy in our study is that the improvement of symptoms such as snoring and mouth breathing was not associated with adenoid size. There might be some association between obstructive symptoms and adenoid size, however, its statistical significance might not be high enough.

Although our study showed the importance of AR after adenotonsillectomy, it has some limitations. First, this is a retrospective study with telephone survey rather than prospective study. Second, most of symptoms were answered by parents of the patients due to their young age. In addition, due to their young age and poor cooperation, it was very difficult to follow up patients 
for long duration that telephone survey was performed. In telephone survey, the information given by respondents could be brief and limited than conventional interview due to time limitation. To overcome these limitations, future studies should address above limitations and should be well-designed prospective studies. In spite of these limitations, this study revealed significant findings that the presence of AR determines postoperative symptoms in adenotonsillectomized patients during long-term follow-up.

Second, we simply divided the patients into AR group and control group. AR group can be subdivided into perennial allergic rhinitis (PAR), seasonal allergic rhinitis (SAR), PAR+SAR. In 131 AR patients, PAR, SAR, and PAR+SAR patients were, 117, 5 , and 9, respectively. Because of the small number of SAR subgroup, subgroup analysis was not performed in our study.

In addition, it would be helpful to include third group of patients who had been treated for AR (medication or immunotherapy) after adenotonsillectomy to prove our hypothesis to evaluate the effect of AR treatment.

In conclusion, this study could suggest that AR is an important risk factor on symptom improvement after adenotonsillectomy. Hence, it is necessary to evaluate the presence of AR in patients scheduled for adenotonsillectomy, and the patients with AR should be taken care of on a long-term basis after adenotonsillectomy.

\section{CONFLICT OF INTEREST}

No potential conflict of interest relevant to this article was reported.

\section{ACKNOWLEDGMENTS}

This study was supported by Dankook University Research Fund, Cheonan, Republic of Korea, in 2015 (by Ji-Hun Mo).

\section{REFERENCES}

1. Mitchell RB. Adenotonsillectomy for obstructive sleep apnea in children: outcome evaluated by pre- and postoperative polysomnography. Laryngoscope. 2007 Oct;117(10):1844-54.

2. Mitchell RB, Kelly J, Call E, Yao N. Long-term changes in quality of life after surgery for pediatric obstructive sleep apnea. Arch Otolaryngol Head Neck Surg. 2004 Apr;130(4):409-12.

3. Mitchell RB, Kelly J, Call E, Yao N. Quality of life after adenotonsil- lectomy for obstructive sleep apnea in children. Arch Otolaryngol Head Neck Surg. 2004 Feb;130(2):190-4.

4. Sadeghi-Shabestari M, Jabbari Moghaddam Y, Ghaharri H. Is there any correlation between allergy and adenotonsillar tissue hypertrophy? Int J Pediatr Otorhinolaryngol. 2011 Apr;75(4):589-91.

5. Friedman M, Ibrahim H, Joseph NJ. Staging of obstructive sleep apnea/hypopnea syndrome: a guide to appropriate treatment. Laryngoscope. 2004 Mar;114(3):454-9.

6. Kemaloglu YK, Goksu N, Inal E, Akyildiz N. Radiographic evaluation of children with nasopharyngeal obstruction due to the adenoid. Ann Otol Rhinol Laryngol. 1999 Jan;108(1):67-72.

7. Chung YJ, Cho IK, Lee KI, Bae SH, Lee JW, Chung PS, et al. Seasonal specificity of seasonal allergens and validation of the ARIA classification in Korea. Allergy Asthma Immunol Res. 2013 Mar;5(2): 75-80.

8. Han M, Shin S, Park H, Park KU, Park MH, Song EY. Comparison of three multiple allergen simultaneous tests: RIDA allergy screen, MAST optigen, and polycheck allergy. Biomed Res Int. 2013;2013: 340513.

9. Baldassari CM, Mitchell RB, Schubert C, Rudnick EF. Pediatric obstructive sleep apnea and quality of life: a meta-analysis. Otolaryngol Head Neck Surg. 2008 Mar;138(3):265-73.

10. Friedman M,Wilson M, Lin HC, Chang HW. Updated systematic review of tonsillectomy and adenoidectomy for treatment of pediatric obstructive sleep apnea/hypopnea syndrome. Otolaryngol Head Neck Surg. 2009 Jun;140(6):800-8.

11. Bhattacharjee R, Kheirandish-Gozal L, Spruyt K, Mitchell RB, Promchiarak J, Simakajornboon N, et al. Adenotonsillectomy outcomes in treatment of obstructive sleep apnea in children: a multicenter retrospective study. Am J Respir Crit Care Med. 2010 Sep; 182(5):676-83.

12. Tasker C, Crosby JH, Stradling JR. Evidence for persistence of upper airway narrowing during sleep, 12 years after adenotonsillectomy. Arch Dis Child. 2002 Jan;86(1):34-7.

13. Vargervik K, Miller AJ, Chierici G, Harvold E, Tomer BS. Morphologic response to changes in neuromuscular patterns experimentally induced by altered modes of respiration. Am J Orthod. 1984 Feb;85 (2):115-24.

14. Wright AL, Holberg CJ, Martinez FD, Halonen M, Morgan W, Taussig LM. Epidemiology of physician-diagnosed allergic rhinitis in childhood. Pediatrics. 1994 Dec;94(6 Pt 1):895-901.

15. Bresolin D, Shapiro PA, Shapiro GG, Chapko MK, Dassel S. Mouth breathing in allergic children: its relationship to dentofacial development. Am J Orthod. 1983 Apr;83(4):334-40.

16. Linder-Aronson S. Adenoids: their effect on mode of breathing and nasal airflow and their relationship to characteristics of the facial skeleton and the denition: a biometric, rhino-manometric and cephalometro-radiographic study on children with and without adenoids. Acta Otolaryngol Suppl. 1970;265:1-132.

17. Kim DK, Han DH. Impact of allergic rhinitis on quality of life after adenotonsillectomy for pediatric sleep-disordered breathing. Int Forum Allergy Rhinol. 2015 Aug;5(8):741-6.

18. Lee CH, Kang KT,Weng WC, Lee PL, Hsu WC. Quality of life after adenotonsillectomy for children with sleep-disordered breathing: a linear mixed model analysis. Int J Pediatr Otorhinolaryngol. 2014 Aug;78(8):1374-80. 